\title{
Les Mammomonogamus des Ruminants domestiques et sauvages
}

\author{
par M. GRABER, J. EUZEBY, J. GEVREY et P.-M. TRONCY
}

Laboratoire de Parasitologie, Ecole nationale de vétérinaire de Lyon,

2, quai Chauveau, F. 69009 Lyon,

Institut d'élevage et de médecine vétérinaire des pays tropicaux, Laboratoire de Farcha, Fort-Lamy, République du Tchad

\section{Résumé}

Les auteurs, à partir d'une quarantaine d'exemplaires de Syngames de Bovidés prélevés dans les collections des Ecoles vétérinaires de Lyon et d'Alfort redécrivent Mammomonogamus laryngeus (Railliet, 1899) et Mammomonogamus nasicola (Von Linstow, 1899). Ils indiquent les caractères permettant de différencier les deux espèces, caractères qui reposent sur la structure et la forme de la capsule buccale, la conformation de la bourse caudale du mâle et de la côte dorsale, l'aspect de la queue de la femelle, la taille des parasites et de leurs œufs.

Mammomonogamus - vraisemblablement $M$. nasicola existe également en République Centrafricaine dans la trachée de Syncerus caffer aequinoxialis.

\section{Summary}

From a survey of a 40-odd specimens of Bovine Syngamidae coming from collections of Lyon and Alfort veterinary schools, the authors give a new description of Mammomonogamus laryngeus (Railliet, 1899) and Mammomonogamus nasicola Von Linstow, 1899). They emphasize the specific features of both species concerning: the buccal capsule (shape and structure), the copulatrix bursa (chiefly dorsal ray), the tail of the females, the size of the worms and the egg. These features are quite valuable for the specific identification of Mammomonogamus species.

It is suggested that Mammomonogamus nasicola equally infect Syncerus caffer aequinoxialis (trachea) in Central African Republic. 
L'autopsie d'un jeune Syncerus caffer aequinoxialis (Blyth) de quatre ans abattu le 23 juin 1970 à 60 kilomètres de $\mathrm{Dji}$ (Est de la République centrafricaine) a permis de recueillir dans la trachée un couple de Mammomonogamus dont l'étude nous a amenés à revoir les caractères morphologiques différentiels des Syngames de ruminants, à l'exclusion de Mammomonogamus okapiae des bronches de l'Okapi.

Pour ce faire, quarante parasites ont été examinés. Dix mâles et dix femelles ont été prélevés dans la collection Marotel de l'Ecole Nationale vétérinaire de Lyon (Buffle domestique d'origine indéterminée) et l'autre moitié dans la collection Railliet de l'Ecole Nationale Vétérinaire d'Alfort (1): il s'agit, dans ce cas, des prototypes de Syngamus laryngeus décrits par Railiet en 1899 et provenant du larynx d'un bœuf sacrifié à l'Institut Pasteur de Nha-Trang (Vietnam) par Carré et Fraimbault en janvier 1899.

\section{Description générale.}

Les Syngames de ruminants sont des Strongyloidea appartenant à la famille des Syngamidés. Ils sont actuellement inclus dans le genre Mammomonogamus (Ryzhikov, 1948, 1949).

Leur longueur est comprise entre $3 \mathrm{~mm}$ (2) chez le Buffle domestique et $33 \mathrm{~mm}$ (3)

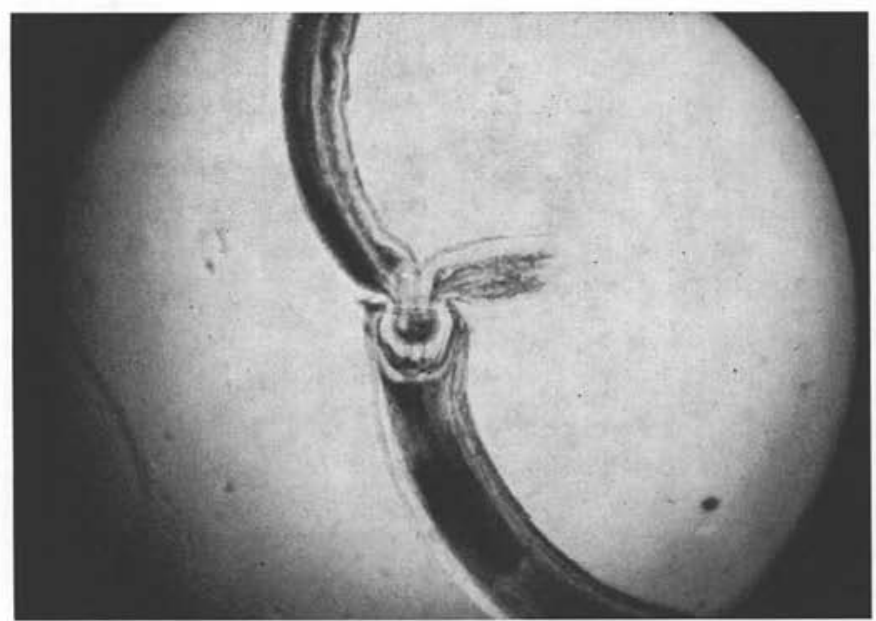

Pното $\mathrm{N}^{\circ}$ I. - Mammomonogamus laryngeus mâle ayant aspiré l'extrémité postérieure de sa planche

(1) L'ensemble de ce matériel était en excellent état de conservation.

(2) Mâle.

(3) Femelle. 
chez diverses Antilopes sauvages (Sachs, Frank et Bindernagel, 1969) et leur largeur eitre $383 \mu$ et $1,2 \mathrm{~mm}$, ce qui leur donne un aspect trapu.

Leur coloration est rouge vif ou blanc marbré et leur cuticule est striée, surtout dans la région antérieure du corps.

Ils sont constamment accouplés et difficiles à séparer. Le mâle est fixé presque à angle droit au quart antérieur du corps de la femelle où se trouve l'orifice vulvaire. La taille des mâles étant sensiblement inférieure à celie des femelles, les extrémités céphaliques du couple sont à peu près au même niveau, ce qui lui donne un aspect caracté-
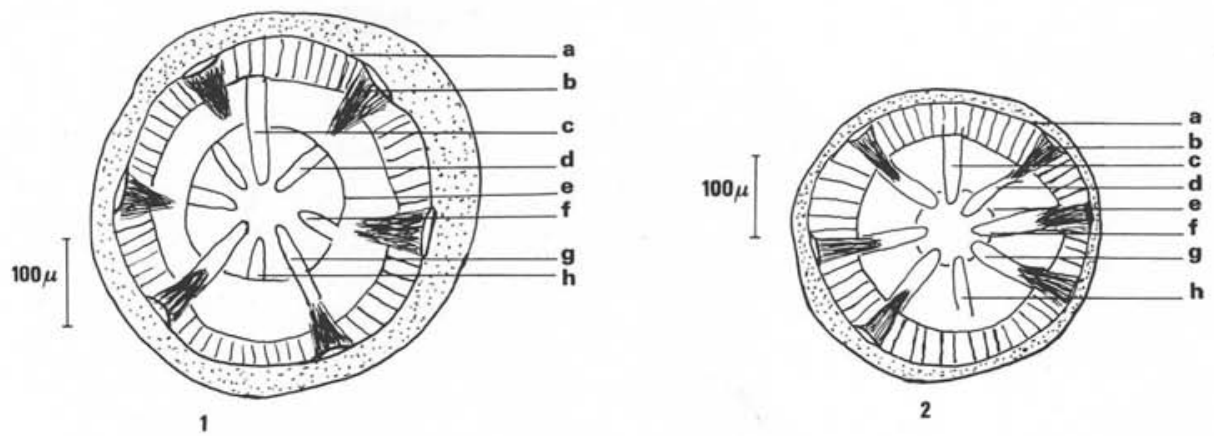

Planche I. - Extrémité antérieure. Vue apicale. 1. Mammomonogamus nasicola (Collection Marotel). 2. Mammomonogamus laryngeus (Collection Railliet): $a$. Paroi de la capsule buccale; $b$. Papilles céphaliques; $c$. Côte dorsale; $d$. Côte sub-dorsale; $e$. Ouverture orale; $f$. Côte latérale; $g$. Côte subventrale; $h$. Côte ventrale

ristique en Y. Parfois, cependant, la position du mâle est complètement inversée, comme le montre la photo $\mathrm{n}^{\circ} 1$, où celui-ci a en quelque sorte «aspiré » une partie de l'extrémité postérieure de sa femelle. Le même phénomène avait déjà été observé par Ware (1925).

L'extrémité antérieure du corps, en vue apicale, a l'aspect suivant: le bord de la capsule est découpé en six endroits différents délimitant six festons inégaux dont les plus longs sont, en principe, en position dorsale et ventrale et les quatre autres en position sub-latérale. Il existe six papilles céphaliques (deux latérales, deux sub-dorsales et deux sub-ventrales) peu visibles, très proches de l'ouverture orale et qui correspondent aux six échancrures de la capsule.

Celle-ci, en vue latérale ou antéro-postérieure (Planches II et III), est petite, épaisse, cupuliforme ou cylindro-conique. A sa base, se trouvent huit dents triangulaires saillantes disposées en cercle, plus ou moins éloignées les unes des autres. De ces dents, partent des côtes chitineuses qui maintiennent l'écartement et la rigidité de la capsule buccale. Certaines d'entre elles traversent toute la paroi de la capsule et aboutissent au 



PlanchB II. - Capsule buccale du mâle. 1. Mammomonogamus laryngeus (Collection Railliet); 2. Mammomonogamus nasicola (Collection Marotel); 3. Mammomonogamus nasicola (Syncerus Caffer-R.C.A.) 

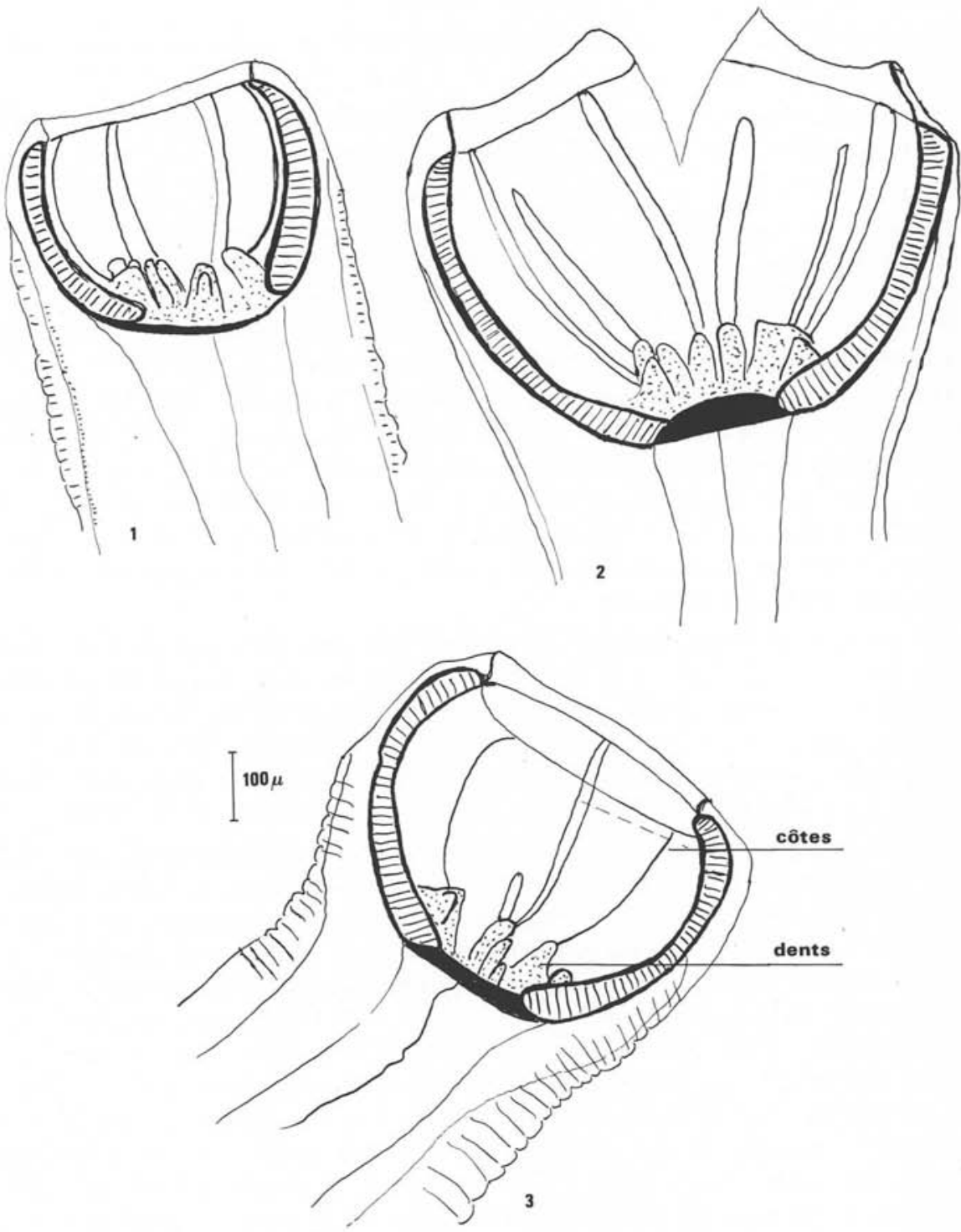

Planche III. - Capsule buccale de la femelle. 1. Mammomonogamus laryngeus (Collection Railliet); 2 Mammomonogamus nasicola (Collection Marotel); 3 Mammomonogamus nasicola (Syncerus Caffer-R.C.A.) 
niveau des échancrures, d'autres s'arrêtent à mi-hauteur. Leur disposition permet de différencier les espèces.

La capsule buccale est suivie d'un œsophage en forme de massue dont la partie antérieure est légèrement dilatée.

Les papilles cervicales arrondies sont, en général, situées au voisinage de l'anneau nerveux, c'est-à-dire à peu de distance du milieu de l'œsophage.

Le mâle est presque cylindrique, un peu plus épais en arrière qu'en avant. La bourse caudale est peu développée. Elle est soutenue par des côtes ventrales souvent réunies en un tronc unique et des côtes latérales très proches les unes des autres, la médio-latérale étant la plus courte. La côte externo-dorsale mince naît séparément de la dorsale. Celle-ci (Planche IV), fendue sur une partie de sa longueur, se termine par deux branches dont l'extrémité est simple ou dichotomisée.

Les cordons testiculaires s'étendent en arrière du milieu du corps. Les spicules, longs de 25 à $30 \mu$ (Chaplin, 1924), ont la forme de fins bâtonnets qu'il est difficile, voire impossible, de mettre en évidence (Railliet, 1899 ; Buckley, 1934) : c'est le cas de tous les mâles ayant fait l'objet de cette étude. De ce fait, ils ne peuvent servir à la diagnose des espèces, comme le suggère Bhalerao (1935). D'après Hall (1920), la régression des spicules et la réduction de la bourse caudale seraient la conséquence de l'accouplement permanent des Syngames.

Le corps de la femelle, 'tronqué à l'extrémité antérieure, s'amincit progressivement à partir de la vulve qui s'ouvre dans le quart antérieur du corps. Le rétrécissement est particulièrement marqué dans la région anale. L'appareil génital est de type prodelphe. La longueur des deux utérus varie en fonction des espèces et des parasites. Les tubes ovariens s'allongent quelquefois fort loin dans la région postérieure, sans jamais atteindre l'anus. La queue (Planche V), courte et rectiligne, se termine en pointe mousse.

Les œufs (Planche VI), dans l'ovojecteur et la terminaison de l'utérus, sont ellipsoïdes, non operculés. Ils sont entourés d'une coque épaisse (4 à 4,6 $\mu$, d'après Teuscher et coll., 1970), jaunâtre, légèrement rugueuse, souvent striée, renfermant deux blastomères qui la remplissent incomplètement. Sur un total de 108 œufs examinés, ces caractères sont rigoureusement constants, quelle que soit l'origine des parasites : seules, les dimensions changent (Tableaux $\mathrm{n}^{\circ *} 1$ et 2 ). Ces observations sont en accord avec celles de Buckley (1934) et de Teuscher et coll. (1970), mais ne correspondent pas à celles de Ware (1925), de Patnaik (1963), de Lévine (1968), de Craig et Faust (1970) : pour ces auteurs, l'œuf de Mammomonogamus laryngeus, au moment de la ponte, comporte de 4 à 8 blastomères, alors que, pour les prototypes de Railliet, il n'en comprend que deux. En réalité, cette différence n'est qu'apparente et n'a pas grande signification en matière de diagnose différentielle. Comme nous avons pu le constater et comme cela a été signalé à propos d'Haemoncus et d'Ostertagia (Gevrey, Communication personnelle), les œufs de Nématodes, dans certaines circonstances, sont susceptibles de poursuivre leur segmentation in utero pendant un temps limité. Tout dépend de la façon dont les Nématodes sont récoltés et conservés. 

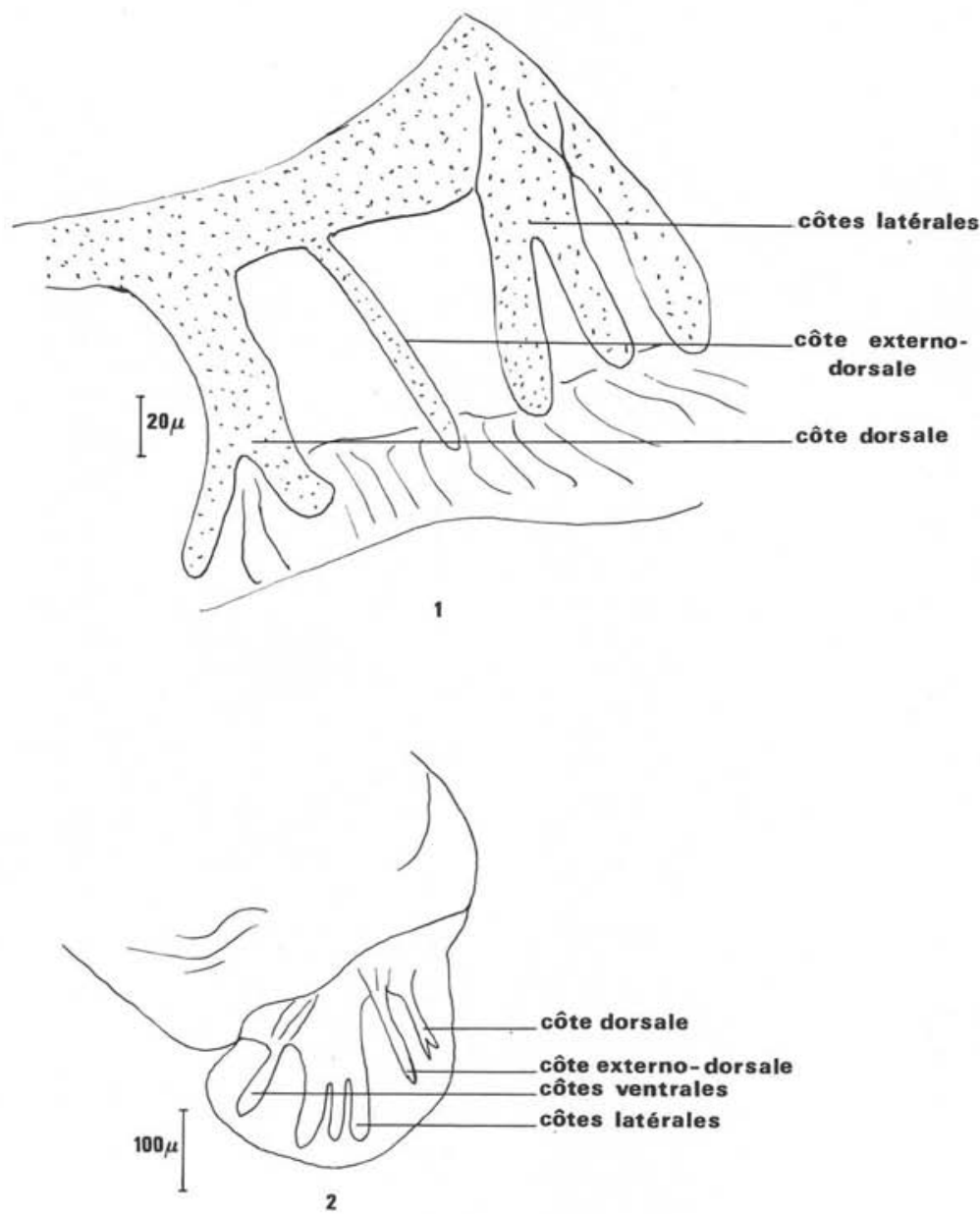

Planche IV. - Bourse caudale du mâle. 1 Mammomonogamus laryngeus (Collection Railliet), côte dorsale; 2 Mammomonogamus nasicola (Syncerus Caffer-R.C.A.), vue latérale 

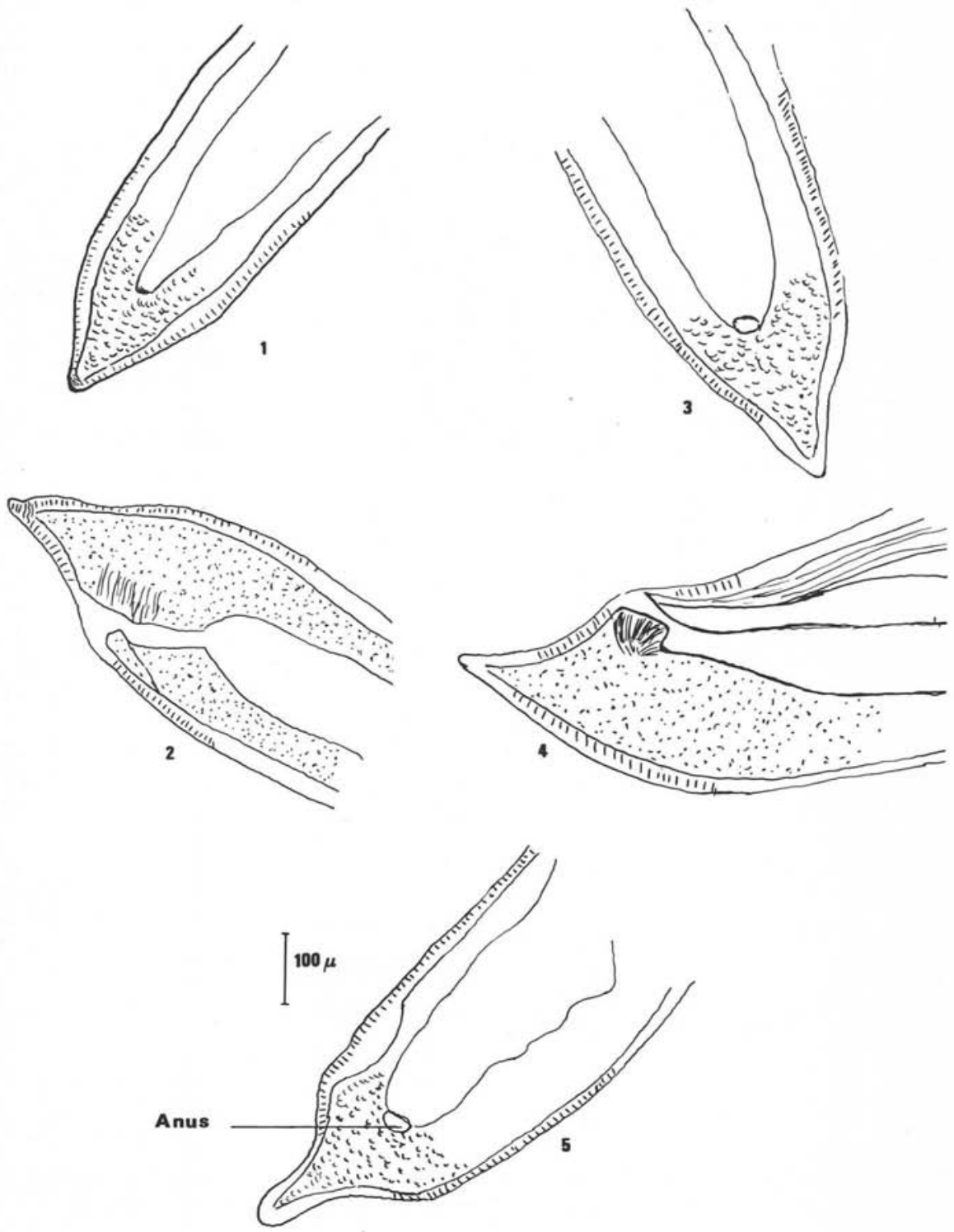

Planche V. - Queue de la femelle. Vue latérale et vue ventrale. 1 et 2 Mammomonogamus laryngeus (Collection Railliet); 3 et 4 Mammomonogamus nasicola (Collection Marotel); 5 Mammomonogamus nasicola (Syncerus Caffer-R.C.A.) 

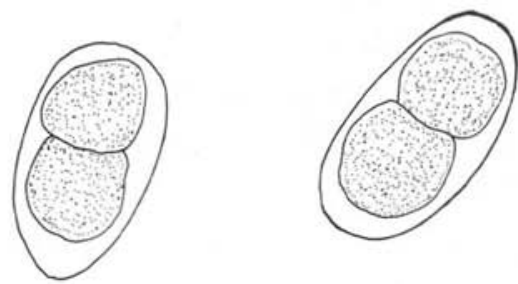

1
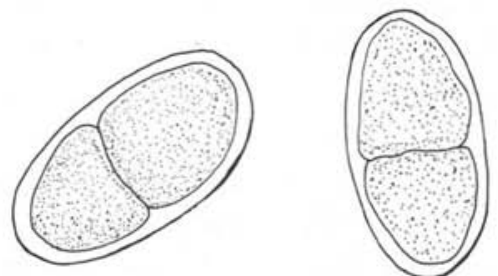

2


$120 \%$

3

Planche VI. - Eufs. 1 Mammomonogamus laryngeus (Collection Railliet); 2 Mammomonogamus nasicola (Collection Marotel); 3 Mammomonogamus nasicolo (Syncerus Caffer-R.C.A.) 


\section{Diagnose différentielle.}

Elle est basée :

- sur les mensurations des parasites et de leurs organes;

- sur certains caractères morphologiques particuliers (Capsule buccale; queue de la femelle et bourse caudale du mâle).

\section{II-1. - Mensurations.}

Elles portent sur la longueur des Nématodes, leur largeur, les dimensions de la capsule buccale, des œufs et de l'œsophage, la position relative de la vulve et de l'extrémité postérieure des circonvolutions utérines.

La moyenne est indiquée avec l'erreur type et la signification de la différence est donnée à $\mathrm{P}<0,05$.

La lecture du tableau $\mathrm{n}^{\circ} 1$ montre qu'il existe des différences de taille assez importantes entre les exemplaires de la collection Railliet et ceux de la collection Marotel, mais que certains critères de diagnose, tels que la situation de la vulve dans la région antérieure du corps et le rapport $\frac{\text { Hauteur }}{\text { Largeur }}$ de la capsule buccale ne sont pas significatifs et ne peuvent donc être pris en considération pour distinguer les espèces. Ces observations recoupent entièrement celles de Buckley (1934).

\section{II.2. - Caractères morphologiques particuliers.}

II.2.1. - La capsule buccale de Mammomonogamus laryngeus de la collection Railliet comporte en profondeur huit dents, d'où partent huit côtes. Six d'entre elles (Pl. I, fig. 2; Pl. II et III, fig. 1), les deux latérales, les deux sub-dorsales et les deux sub-ventrales, atteignent le bord antérieur de la capsule buccale qu'elles divisent en six festons. Les deux dernières côtes, la dorsale et la ventrale, s'arrêtent à mi-chemin ou légèrement au-delà.

Ces caractères sont bien ceux décrits par Sheather et Shilston (1920), Ware (1925) et Buckley (1934) à partir de Syngames de la même espèce originaires des Indes (Muktesar) et de Malaisie. Ils ne correspondent ni à la description originale de Railliet (1899) qui fait état de huit festons et de huit côtes suivant toute la hauteur de la paroi, ni à celle de De Does (1907) qui parle de six festons et de sept côtes.

Les Mammomonogamus de la collection Marotel (Pl. I, fig. 1 ; Pl. II et III, fig. 2) ont également une capsule buccale pourvue de six festons et de huit dents profondes. De celles-ci, partent des côtes dont la disposition, la plupart du temps, est la suivante : 


\begin{tabular}{|c|c|c|c|c|c|c|c|}
\hline \multicolumn{2}{|c|}{ 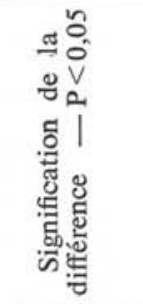 } &  & 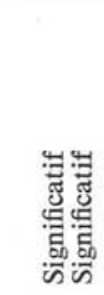 & 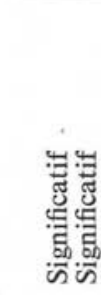 & 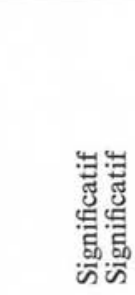 & 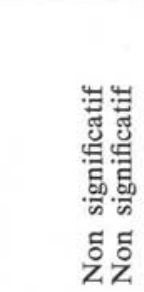 & 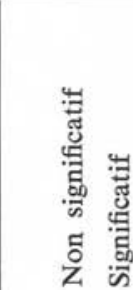 \\
\hline 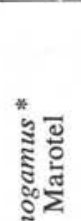 & 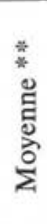 &  & 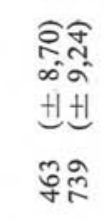 &  & 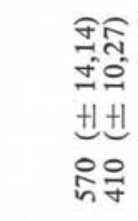 &  &  \\
\hline 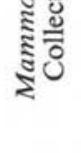 & 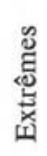 &  & 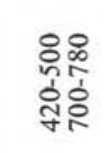 & 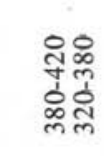 &  & $\begin{array}{l}80 \\
0 \\
0 \\
0 \\
0 \\
0 \\
0 \\
0\end{array}$ & 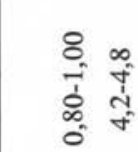 \\
\hline 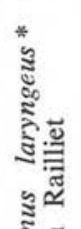 & 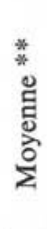 & 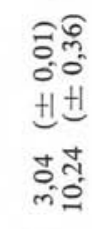 &  & 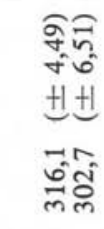 &  & 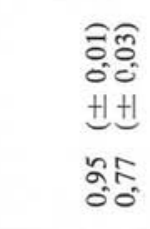 & 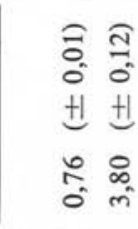 \\
\hline  & 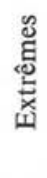 &  & 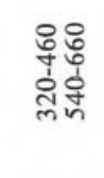 & 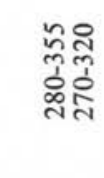 &  & $\begin{array}{l}8 \overline{0} \\
-0 \\
010 \\
0 \\
0 \\
0 \\
0 \\
0\end{array}$ & 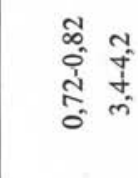 \\
\hline & & 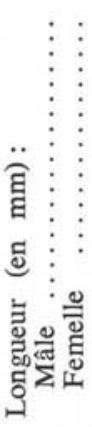 & 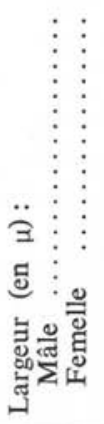 & 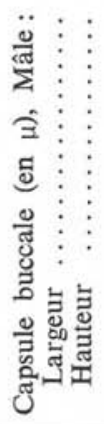 & 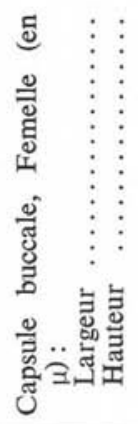 &  & 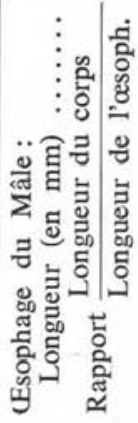 \\
\hline
\end{tabular}


M. GRABER, J. EUZEBY, J. GEVREY ET P. M. TRONCY

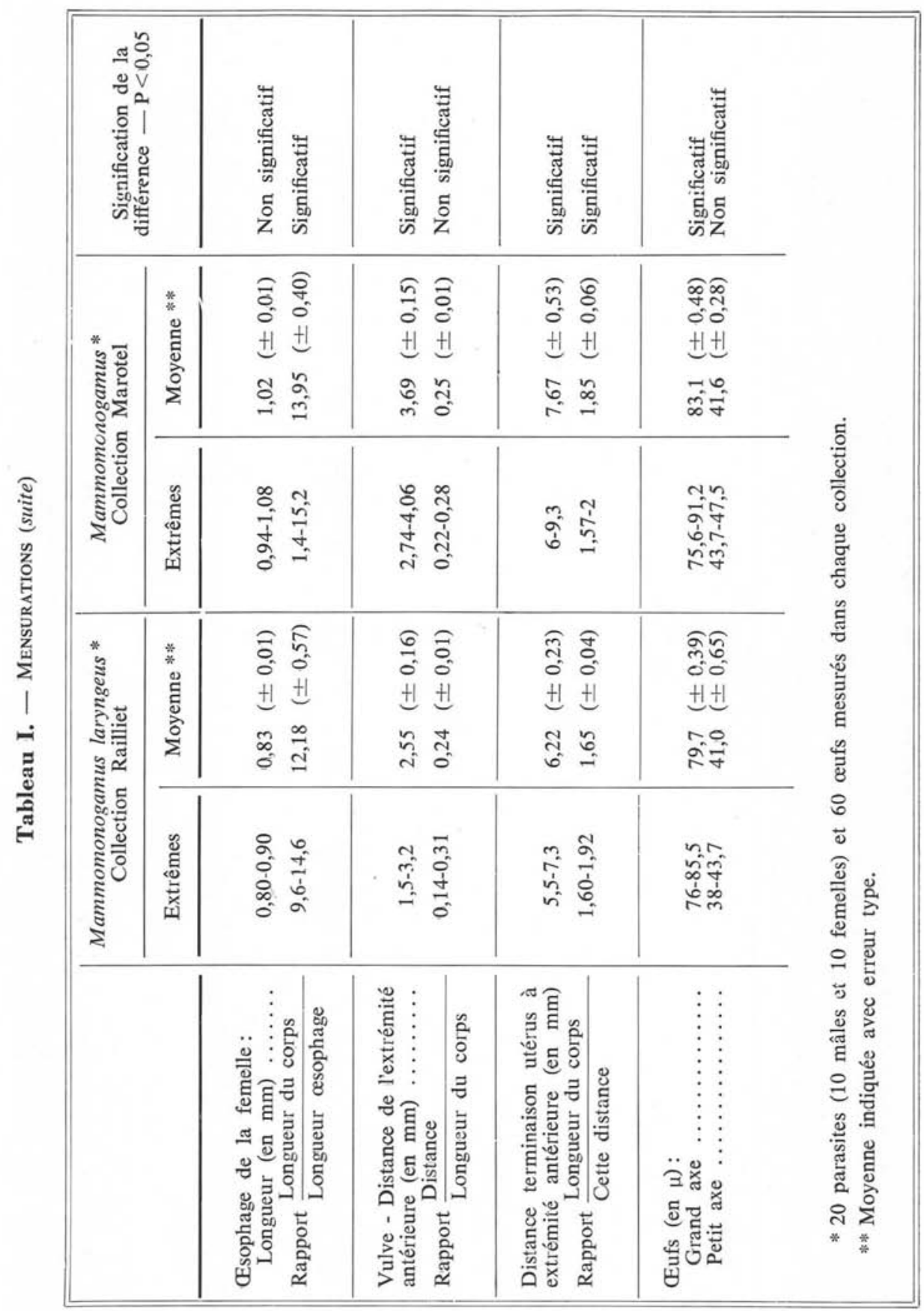


la dorsale et les deux sub-ventrales sont complètes et longent la paroi de la capsule de bout en bout; les autres se terminent à des hauteurs variables (moitié ou deux-tiers).

II.2.2. - La bourse caudale des mâles de Mammomonogamus laryngeus et de Mammomonogamus de la collection Marotel présentent des différences minimes qui intéressent la côte dorsale : dans le premier cas, les extrémités de chaque branche sont simples (Pl. IV, fig. 1), tandis que dans le second cas, elles sont divisées en deux (Pl. IV, fig. 2). La mise en évidence de ces détails est particulièrement laborieuse.

II.2.3. - La queue de la femelle de Mammomonogamus laryngeus (Pl. V, fig. 1 et 2) est courte, obtuse et montre de nombreuses stries très fines. La distance de l'anus à l'extrémité postérieure est comprise entre 173,3 et 236,7 $\mu$ (en moyenne, 204,2 $\mu$ ).

Chez les Mammomonogamus de la collection Marotel, la queue est un peu plus longue $(213-320 \mu$, en moyenne $254 \mu$ ), plus pointue et dépourvue de stries.

\section{Discussion.}

Il apparaît donc nettement que les Mammomonogamus de la collection Marotel, quoique très proches de Mammomonogamus laryngeus, constituent une espèce différente que l'on peut assimiler à Mammomonogamus nasicola. Ce Syngame a été sommairement décrit par Von Linstow en 1899 à partir d'exemplaires recueillis dans les cavités nasales d'une chèvre à Yaoundé (République fédérale du Cameroun) et dans le larynx de Cervus rufus au Brésil (Rio Grandé Do Sul). Plus tard (1934), le matériel d'origine a été réexaminé par Buckley qui n'observe, à l'intérieur de la capsule buccale que trois côtes complètes, au lieu des six indiquées par Linstow, erreur qui «ipso facto » entraîne la mise en synonymie avec Mammomonogamus laryngeus.

Depuis, Mammomonogamus nasicola a été retrouvé à de nombreuses reprises chez le mouton et chez le bœuf aux Antilles (Ste-Lucie, St-Vincent). Il existerait également en Equateur (Teuscher et Coll., 1970).

Il peut aussi passer chez l'homme : connu d'abord sous le nom de Syngamus kingi (Leiper, 1913), le parasite a été confondu avec Mammomonogamus nasicola par Buckley (1934).

Le tableau $\mathrm{n}^{\circ} 2$ donne les dimensions comparées de Mammomonogamus nasicola faites par les différents auteurs qui ont eu la possibilité d'étudier ce Nématode.

Quant aux Syngames du buffle de R.C.A., de par leurs dimensions (Tableau $\mathrm{n}^{\circ}$ 2) et leurs caractères morphologiques (Pl. II et III, fig. 3 ; Pl. V, fig. 5 ; Pl. VI, fig. 3), ils semblent s'apparenter étroitement à Mammomonogamus nasicola: seule, la vulve est située en position plus antérieure. L'examen n'ayant porté que sur un couple, il est difficile, pour l'instant, de tirer des conclusions définitives.

Il est bon de souligner, à ce propos, que la Mammomonogamose du buffle sauvage (Syncerus caffer) n'est pas inconnue en Afrique noire et que le premier cas observé l'a été par Sachs, Frank et Bindernagel (1969) à la réserve Aswa Lolim, dans le Nord- 
M. GRABER, J. EUZEBY, J. GEVREY ET P. M. TRONCY

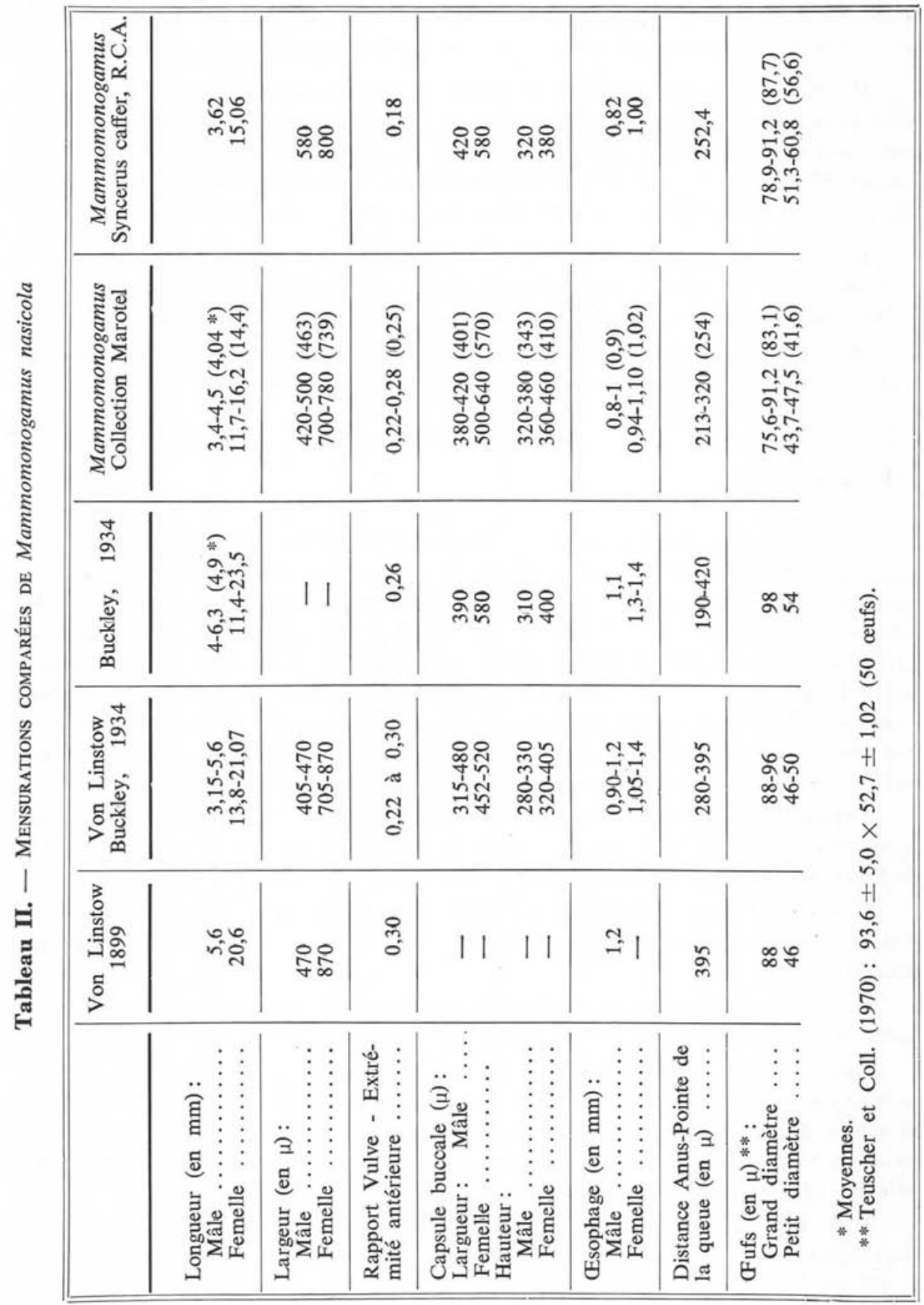


Est de l'Uganda. D'autres Syngames, non encore identifiés, ont également été découverts par les mêmes auteurs dans les cavités nasales du Waterbuck et de divers Kob (Kobus vardoni et Kobus Kob), en Tanzanie et en Uganda.

Mammomonogamus nasicola est différent des autres Mammomonogamus de la grande faune africaine actuellement connus, c'est-à-dire $M$. hippopotami (Gedoelst, 1924) de l'Hippopotame, M. loxodontis (Vuylsteke, 1935) de l'éléphant et $M$. okapiae (Van den Berghe, 1937 ; Baer, 1950) de l'Okapi : il y a divergence de dimensions et d'hôtes.

En revanche, la distinction entre Mammomonogamus laryngeus et Mammomonogamus nasicola est délicate et n'a pas toujours été faite avec précision. Les descriptions originales de Railliet et de Von Linstow en 1899 ne font pas ressortir avec suffisamment de clarté certains caractères essentiels, comme celui de la capsule buccale. Des confusions se sont peu à peu glissées (Ware, 1925; Vaz, 1935), et les deux espèces ont finalement été mises en synonymie par Ryzhikov (1948) et par Skjabin et coll. (1961). Cette manière de voir est, cependant, loin d'être partagée par tous les auteurs (Neveu-Lemaire, 1936; Yamaguti, 1961 ; Gretillat, 1966 ; Lapage, 1967 ; Lévine, 1968).

Le réexamen des prototypes de Railliet montre que Mammomonogamus laryngeus présente un certain nombre de caractères bien particuliers, à savoir :

- La disposition et la hauteur des côtes qui soutiennent la capsule buccale.

- La forme de celle-ci (cyclindro-conique chez le mâle).

- L'aspect de la bourse caudale et des branches qui terminent la côte dorsale (non dichotomisées).

- La queue de la femelle, courte, obtuse et légèrement striée.

- Les œufs à deux blastomères qui mesurent 76-85 $\mu$ sur 38-47 $\mu$ (contre 76$98 \mu \times 41-56 \mu$ pour Mammomonogamus nasicola).

- Les dimensions du parasite et des organes qu'il renferme : en général, comme le confirment Hall (1915) et Buckley (1934) sur des exemplaires provenant des Philippines et de Malaisie, Marnmomonogamus laryngeus est un petit Nématode dont la taille est comprise entre $2,7 \mathrm{~mm}$ (mâle) et $11,7 \mathrm{~mm}$ (femelle). Toutefois, plusieurs auteurs (Ware, 1925; Patnaik, 1963) donnent des longueurs bien supérieures: 6,5 à 22,6 mm chez la femelle et 2,5-5,5 mm chez le mâle et Smit (1922), devant cette situation, a cru bon de proposer deux sous-espèces, $M$. laryngeus minor et $M$. laryngeus major. Les descriptions étant souvent incomplètes, il est difficile de savoir si l'on a bien affaire, dans tous les cas, à Mammomonogamus laryngeus.

- La répartition géographique: Mammomonogamus laryngeus a surtout été rencontré dans le Sud-Est asiatique et Mammomonogamus nasicola en Afrique, en Amérique du Sud et aux Antilles.

En définitive, les Mammomonogamus des ruminants domestiques et sauvages comprennent actuellement trois espèces :

M. okapiae de l'Okapi. 
$M$. laryngeus du buffle, du bœuf et du zébu.

M. nasicola du bœuf, du mouton, de la chèvre, de Cervus rufus et de Syncerus caffer.

Ces deux dernières sont morphologiquement très voisines : seules, des études plus précises de biologie permettront ou non de les mettre en synonymie.

\section{REMERCIEMENTS}

Les auteurs tiennent à remercier vivement M. le Professeur Guilhon qui a bien voulu mettre à leur disposition les exemplaires de $M$. laryngeus de la collection Railliet.

\section{Bibliographie}

BAER (J.-G.), 1950. - Etude critique des parasites de l'Okapi. Acta. Trop., 7, 2, 164-192. Bhalerao (G. D.), 1935. - Helminth parasites of the domestic animals in India. Monographie, $\mathrm{n}^{\circ} 7,287$.

BUCKLeY (J. J. C.), 1934. - On Syngamus nasicola Linstow, 1899, from sheep and cattle in the West Indies. J. Helminth., 12, 1, 47-62.

Chapin (E. A.), 1924. - The spicules of Syngamus laryngeus, Parasit., 11, 106.

-, 1925. - Review of the Nematode genera Syngamus Sieb. and Cyathostoma. J. Agric. Res., 30, 557-70.

DE Does (K. J.), 1907. - Berchrijning van eenige dierlijke parasitien. Geneesk. Tijdschr. Ner-Ind., 28, 263-79.

Froger (P.-E.-J.), 1930. - La Syngamose. Thèse vétérinaire, Paris, 62 pp.

Gedoelst (L.), 1924. - Un Syngame, parasite de l'Hippopotame. Annls Parasit. Hum. Comp., 2, 4, 307-11.

Gretillat (S.), 1966. - Mission I.E.M.V.T.-I.N.R.A. Antilles françaises, 34-7.

Hall (M. C.), 1915. - A note on Syngamus laryngeus from cattle in the Philippine islands. Am. J. Vet. Med., 10, 395-6.

-, 1920. - Apparent atrophy of spicules with increasingly close and permanent union of the male and the female Syngamus. Parasit., 7, 100.

Lapage (G.), 1967. - Veterinary Parasitology. Oliver Boyd Edimburg and London, 139.

LeIPeR (R. T.), 1913. - Observations on certain Helminths of man. Trans. R. Soc. Trop. Med. Hyg., 6, 8, 265-97.

-, 1913. - Gapes in man, on occasional helminthic infection. Lancet, 10, 170.

Lent (H.) and Penna (M.), 1939. - Novo caso de Singamose em homon no Brasil. Hospital, Rio de J., 16, 1, 111-18.

Levine (N. P.), 1968. - Nematode parasites of domestic animals and of man. Burg. Pub. Comp., 157-8.

Linstow (O. Von), 1899. - Nematoden aus der Berliner zoologischen sammlung. Mitt. Zool. Mus. Berl., 1, 5-26. 
Neveu-Lemaire (M.), 1936. - Traité d'Helminthologie médicale et vétérinaire. Vigot frères, Paris, 974-5.

Patknaik (M. M.), 1963. - A note on bovine Syngamosis. Ind. Vet. J., 40, 272-4.

Railliet (A.), 1899. - Syngame laryngien du bœuf. C.R. Séanc. Soc. Biol., 6, 10, 174-76.

RyzHIKov (K. M.), 1948. - Phylogenic relationship of Nematodes of the family Syngamidae and an attempt to reconstruct their systematics (en russe). Dokl. Akad. Nauk. S.S.S.R., 62, 5, 733-36.

—, 1949. - Principles of Nematodology. Vol. I : Syngamidae of domestic and wild animals (en russe). Izcat. Akad. Nauk. S.S.S.R., Leningrad, Moscow, 164 pp.

Sachs (R.), Frank (H.) and Bindernagel (J. A.), 1969. - New host records for Mammomonogamus in African game animals through application of a simple method of collection. Vet. Rec., 84, 22, 562-3.

SheAther (A. L.) and Shilston (A. W.), 1920. - Syngamus laryngeus in cattle and buffaloes in India. Bull. Agric. Res. Pusa, 92, 1-8.

Skrjabin (K. I.), Shikhobalova (N. P.), Schultz (R. S.), Popova (T. I.), Boev (S. N.) and Delyamure (S. L.), 1961. - Key to parasitic Nematodes. Vol. III : Strongylata. Israël Prog. Trans. Jerusalem, 187-190.

SмIт (H. J.), 1922. — Parasitologische studien in Nierderlândisch-Indien. Dt. Tierärztl. Wschr., 30, 506-12; 526-31; 546-48.

Teuscher (E.), Komijin (R. E.), Robalino (R.) et SMith (R. D.), 1970. - Le diagnostic coprologique de la Syngamose bovine en Equateur avec une note sur l'efficacité probable du Tétramisole. Zentbl. Vét. Méd., 17, 2, 324-30.

THéry (A.), 1962. - Les Syngamoses. Thèse vétérinaire, Paris, 74 pp.

VAN DEN Berghe (L.), 1937. - Contribution à l'étude des parasites de l'Okapi. Rev. Zool. Bot. Afr., 29, 141-50.

VUYLSTEKe (C.), 1937. - Etude de quelques Nématodes, parasites de l'éléphant. Rev. Zool. Bot. Afr., 27, 323-25.

VAZ (Z.), 1935. - Sobre a pretensa validez de Syngamus nasicola. Presence do Syngamus laryngeus nas fossas nasaes de carneiros do Brasil. Archos. Inst. Biol. S.-Paulo, 6, 35-39.

Ware (F.), 1925. - Two uncommon Nematode parasites of cattle. J. Comp. Path., 38, 2, 86-89.

Yamaguti (S.), 1961. - Systema helminthum. Vol. III: The Nematodes of vertebrates, Part I, 531-2. 